\title{
Potent therapeutic activity of folate receptor-targeted liposomal carboplatin in the localized treatment of intraperitoneally grown human ovarian tumor xenograft
}

\author{
This article was published in the following Dove Press journal: \\ International Journal of Nanomedicine \\ 13 February 2012 \\ Number of times this article has been viewed
}

\author{
Anumita Chaudhury' \\ Surajit Das' \\ Ralph M Bunte ${ }^{2}$ \\ Gigi NC Chiu' \\ 'Department of Pharmacy, Faculty \\ of Science, National University \\ of Singapore, ${ }^{2}$ Duke-NUS Graduate \\ Medical School, Singapore, \\ Republic of Singapore
}

Correspondence: Gigi NC Chiu Department of Pharmacy, Faculty of Science, National University of Singapore, I8 Science Drive 4, Singapore II 7543

Tel +6565165536

Fax $+656779 I 554$

Email phacncg@nus.edu.sg

\begin{abstract}
Intraperitoneal (IP) therapy with platinum (Pt)-based drugs has shown promising results clinically; however, high locoregional concentration of the drug could lead to adverse side effects. In this study, IP administration was coupled with a folate receptor-targeted (FRT) liposomal system, in an attempt to achieve intracellular delivery of the Pt-based drug carboplatin in order to increase therapeutic efficacy and to minimize toxicity. In vitro and in vivo activity of FRT carboplatin liposomes was compared with the activity of free drug and nontargeted (NT) carboplatin liposomes using FR-overexpressing IGROV-1 ovarian cancer cells as the model. Significant reduction in cell viability was observed with FRT liposomes, which, compared with the free drug, provided an approximately twofold increase in carboplatin potency. The increase in drug potency was correlated with significantly higher cellular accumulation of Pt resulting from FRT liposomal delivery. Further evaluation was conducted in mice bearing intraperitoneally inoculated IGROV-1 ovarian tumor xenografts. A superior survival rate (five out of six animals) was achieved in animals treated with FRT carboplatin liposomes, injected intraperitoneally with a dose of $15 \mathrm{mg} / \mathrm{kg}$ and following a schedule of twice-weekly administration for 3 weeks. In contrast, no survivors were observed in the free drug or NT carboplatin liposome groups. The presence of cancer cells in lung and liver tissues was observed in the saline, free carboplatin, and NT carboplatin liposome groups. However, there was no sign of cancer cells or drugrelated toxicity detected in tissues from the animals treated with FRT carboplatin liposomes. The results of this study have demonstrated for the first time that the approach of coupling IP administration with FRT liposomal delivery could provide significantly improved therapeutic efficacy of carboplatin in the treatment of metastatic ovarian cancer.
\end{abstract}

Keywords: liposomes, ovarian cancer, targeted therapy, FRT carboplatin liposomes

\section{Introduction}

Poor prognosis and fewer treatment options make advanced ovarian cancer, one of the leading causes of cancer-related death in women, difficult to treat. ${ }^{1,2}$ Inadequate drug concentration at the tumor site is the major cause of treatment failure in solid tumors of peritoneal origin. ${ }^{3,4}$ Drug administration through the intraperitoneal (IP) route could provide a number of benefits: (1) the drug is administered directly to the site of disease, the peritoneal cavity; (2) IP administration could achieve a higher locoregional drug concentration and a longer half-life of a drug in the peritoneal cavity than the intravenous route; and (3) absorption from the peritoneal cavity into the systemic circulation could expose the extraperitoneal sites of metastasis to the admin- 
istered drug. ${ }^{5}$ The proof of concept for IP chemotherapy in ovarian cancer patients was established by a randomized phase III clinical trial (Protocol 172) from the Gynecologic Oncology Group, showing a superior survival benefit in patients with stage III ovarian cancer when treated with IP cisplatin followed by paclitaxel after primary surgical tumor debulking. ${ }^{6}$ Several other studies have also demonstrated the significant benefit of IP chemotherapy over the intravenous route in ovarian cancer patients. ${ }^{7,8}$ Based on these clinical studies, the US National Cancer Institute has recommended IP chemotherapy for the treatment of advanced-stage ovarian cancer.

Platinum (Pt)- and taxane-based drugs constitute the first-line chemotherapy treatment in ovarian cancer and therefore exploration with IP chemotherapy is mostly attempted with these two families of drugs. While IP chemotherapy offers a significant survival benefit in ovarian cancer patients, this method of treatment has not been widely adopted in the clinic; this is because of scrutiny of some of the clinical study designs that included a number of variables changed at one time, including dose, frequency, and route of administration, making clinical adoption more challenging. IP administration of chemotherapy is also faced with various issues. In addition to the need for experienced personnel and facilities for the management of initial side effects such as abdominal discomfort and neuropathy from IP cisplatin administration, ${ }^{9}$ rapid absorption of the drugs into the systemic circulation or poor absorption/penetration into solid tumors also compromise the effectiveness of the IP drug treatment. ${ }^{8,10,11}$ Therefore, further improvements to the IP method of drug administration are necessary.

In this study, IP drug administration is coupled with an ovarian cancer cell targeted liposomal system to achieve intracellular delivery of the Pt-based drug carboplatin, so as to further increase the therapeutic efficacy of localized chemotherapy. Recently, a number of clinical trials have indicated the benefit of using IP carboplatin compared with IP cisplatin, because of the different toxicity profiles with reduced abdominal discomfort and neuropathy. ${ }^{12-16}$ Therefore, the targeted IP administration of carboplatin for the treatment of ovarian cancer should be explored. It is established that over $90 \%$ of epithelial ovarian cancers demonstrate overexpression of folate receptor (FR), which is minimally expressed in normal tissues or organs. ${ }^{17,18}$ Anticancer therapy targeting the FR is further supported by the recent phase I clinical trial of farletuzumab, a humanized monoclonal antibody against FR-alpha that has shown promising therapeutic effects. ${ }^{18,19}$ Therefore, a drug delivery system such as the liposomal system, with a good drug payload designed to target the FR, could be used as the enabling technology to deliver carboplatin into ovarian cancer cells. The authors developed a FR-targeted (FRT) liposomal formulation for carboplatin and compared this with the free drug and nontargeted (NT) liposome formulation under in vitro and in vivo settings. The results of this study have demonstrated for the first time that IP administration of carboplatin-loaded FRT liposomes could produce significant improvement in the therapeutic efficacy of carboplatin in a FR-overexpressing, IP IGROV-1 ovarian tumor xenograft model.

\section{Materials and methods Materials}

Supplies of 1,2-dipalmitoyl-sn-glycero-3-phosphatidylcholine (DPPC), 1,2-distearoyl-sn-glycero-3-phosphatidylcholine (DSPC), 1,2-distearoyl-sn-glycero-3-phosphoethanolamine$\mathrm{N}$-[methoxy(polyethylene glycol)- $\left.{ }_{1000}\right]$ (ammonium salt) (DSPE-PEG ${ }_{1000}$ ), and 1,2-distearoyl-sn-glycero-3-phosphoethanolamine-N-[folate(polyethylene glycol)- ${ }_{2000}$ ] (ammonium salt) (DSPE-PEG ${ }_{2000}$-folate) were purchased from Avanti Polar Lipids, Inc (Alabaster, AL). Supplies of 3-(4,5-dimethylthiazoly-2)-2,5-diphenyltetrazolium bromide (MTT) and dimethyl sulfoxide were purchased from MP Biomedicals LLC (MP Biomedicals Asia Pacific, Singapore). Chloroform was obtained from Merck and Co, Inc (Whitehouse Station, NJ). Carboplatin injection was purchased from Teva Pharmaceuticals Industries Ltd (Petah Tikva, Israel) through the pharmacy of the National University Hospital, Singapore. Carboplatin (powdered drug), 10\% buffered formalin solution, glacial acetic acid, crystal violet, Pt standard solution for atomic absorption spectroscopy (AAS), Sephadex ${ }^{\circledR}$ G-50, and all other reagents were purchased from Sigma-Aldrich Co, LLC (St Louis, MO).

\section{Mice and tumor cell line}

Six- to eight-week-old female CB-17 severe combined immunodeficiency mice were purchased from Charles River Laboratories International, Inc (Wilmington, MA). The animals were housed in microisolator cages in a pathogen-free animal bio-safety level-2 facility. Mice were maintained on a folate-free diet (Dyets, Inc, Bethlehem, PA) 2 weeks before and during the experiment. All procedures involving the use and care of mice were approved by the Institutional Animal Care and Use Committee of the National University of Singapore and were performed in line with the National Advisory Committee for Laboratory Animal 
Research guidelines. The FR-positive human ovarian cancer cell line IGROV-1 was a gift from Professor Larry H Matherly, Wayne State University School of Medicine, Detroit, MI. The cell line was cultured in folate-free RPMI 1640 media (Gibco, Grand Island, NY), supplemented with $100 \mathrm{U} / \mathrm{mL}$ penicillin, $100 \mu \mathrm{g} / \mathrm{mL}$ streptomycin, and $10 \%$ fetal bovine serum (FBS; HyClone Laboratories, Inc, Logan, UT) at $37^{\circ} \mathrm{C}$ in a humidified atmosphere containing $5 \% \mathrm{CO}_{2}$.

\section{Liposome making and drug loading}

Liposome formulations were prepared according to an established procedure. ${ }^{20}$ The following lipid compositions were used for the different liposomal formulations. For NT liposomes, the compositions were DPPC/DSPE-PEG ${ }_{1000}$ or DSPC/DSPE-PEG ${ }_{1000}$ in a molar ratio of 95:5, and are denoted as DPPC-NT and DSPC-NT, respectively. For FRT liposomes, the compositions were DPPC/DSPE$\mathrm{PEG}_{1000} / \mathrm{DSPE} \mathrm{PEG}_{2000}$-folate or DSPC/DSPE-PEG ${ }_{1000} /$ DSPE-PEG $_{2000}$-folate in a molar ratio of 95:4.8:0.2, and are denoted as DPPC-FRT and DSPC-FRT, respectively. Briefly, lipids in appropriate proportions were dissolved in chloroform and the mixture was subsequently dried under a stream of nitrogen gas to make a fluffy lipid film. The sample was placed under vacuum to remove residual solvent. The resultant lipid film was hydrated with $0.9 \%$ sodium chloride ( $\mathrm{pH} \mathrm{7.5)} \mathrm{for} 1$ hour with continuous stirring, and it was subsequently extruded ten times using an extruder (Thermobarrel Extruder; Northern Lipids Inc, Vancouver, $\mathrm{BC}$, Canada) through stacked $100 \mathrm{~nm}$ polycarbonate membranes. Both hydration and extrusion steps were performed at a temperature of $55^{\circ} \mathrm{C} \pm 2^{\circ} \mathrm{C}$.

Carboplatin was encapsulated in liposomes according to the passive equilibration method. ${ }^{20}$ Briefly, the extruded liposome sample was added to carboplatin powder at a drug-to-lipid (D/L) weight ratio of $0.25: 1$ and incubated at $55^{\circ} \mathrm{C} \pm 2{ }^{\circ} \mathrm{C}$ with gentle stirring for 1 hour. At specified time points, an aliquot of the drug-liposome mixture was passed down a $1 \mathrm{~mL}$ Sephadex G-50 spin column pre-equilibrated with $0.9 \%$ saline ( $\mathrm{pH} 7.5$ ) to separate unincorporated carboplatin from the liposomes, with centrifugation at $680 \times \mathrm{g}$ for 3 minutes. All liposomal samples were stored at $2{ }^{\circ} \mathrm{C}-8^{\circ} \mathrm{C}$ until required. The mean diameter (size) and polydispersity index of empty and drug-loaded liposomes were determined at $632 \mathrm{~nm}$, using a Zetasizer ${ }^{\circledR}(3000 \mathrm{HS}$; Malvern Instruments Ltd, Worcestershire, UK).

To determine the final $\mathrm{D} / \mathrm{L}$ weight ratio and encapsulation efficiency, carboplatin and liposomal lipid concentrations were determined. Briefly, carboplatin concentration was analyzed in duplicate by AAS using a spectrometer (PerkinElmer AAnalyst 100; PerkinElmer Inc, Waltham, MA) attached to a graphite tube atomizer, according to an established procedure. ${ }^{20}$ Samples were diluted with $1 \%$ nitric acid to yield a final Pt concentration of 50-250 ng/mL. The analysis was performed at a wavelength of $265.9 \mathrm{~nm}$, and the temperature was calibrated to be $90^{\circ} \mathrm{C}$ for 30 seconds, $120^{\circ} \mathrm{C}$ for 10 seconds, $1100^{\circ} \mathrm{C}$ for 15 seconds, $2700^{\circ} \mathrm{C}$ for 5 seconds (reading time), and $30^{\circ} \mathrm{C}$ for 40 seconds. A standard curve was plotted using a Pt standard solution. The Fiske and Subbarow ${ }^{21}$ phosphate assay method was used to determine liposomal lipid concentrations.

\section{Cryogenic transmission electron microscopy}

Morphological features of empty and drug-loaded FRT liposomes were examined through cryogenic transmission electron microscopy (cryo-TEM). Briefly, diluted liposome samples were placed onto a copper grid $(-3.8 \mu \mathrm{L})$ and blotted to form a thin aqueous layer. Subsequently, the sample was flash-frozen in liquid ethane and transferred to a cryo-TEM grid holder precooled and maintained in liquid nitrogen. The cryo-TEM specimen holder was subsequently transferred to a transmission electron microscope (JEM-2010F; Japan Electron Optics Laboratory Co, Ltd, Tokyo, Japan) for analysis at a voltage of $120 \mathrm{kV}$.

\section{In vitro drug release}

The release of carboplatin from liposomes was determined by dialyzing $0.5 \mathrm{~mL}$ samples ( $1 \mathrm{mg} / \mathrm{mL}$ lipid concentration) in mini dialysis cassettes (Thermo Fisher Scientific Inc, Rockford, IL; molecular weight cutoff $3.5 \mathrm{~K}$ ) against $0.5 \mathrm{~L}$ of phosphate-buffered saline (PBS, $\mathrm{pH} 7.4$ ) at $37^{\circ} \mathrm{C}$ for 72 hours with constant stirring. Separate dialysis cassettes were used for each time point. For release in serum-containing medium, drug-loaded liposomes were mixed with an equal volume of FBS and incubated at $37^{\circ} \mathrm{C}$ over 48 hours. At specified time points, aliquots of the mixture were taken and passed down a $1 \mathrm{~mL}$ Sephadex G-50 spin column. Carboplatin and liposomal lipid concentrations were subsequently determined as previously described.

\section{Cellular accumulation of liposomal carboplatin}

Fluorescent liposomes were prepared for the study of the cellular accumulation of liposomal carboplatin. Both NT 
and FRT liposomes were labeled with $0.5 \mathrm{~mol} \% \mathrm{DiI}$, and all the steps were carried out either in the dark or by wrapping the samples in aluminum foil. IGROV- 1 cells $\left(1 \times 10^{6}\right)$ were plated in $35 \mathrm{~mm}$ dishes and allowed to attach overnight. After 24 hours, $250 \mu \mathrm{M}$ of free carboplatin or carboplatin-loaded NT or FRT liposomes was added to the cells and they were incubated for 24 hours at $37^{\circ} \mathrm{C}$. At the end of the incubation period, the cells were washed three times with cold PBS to remove the unbound liposomes, and they were visualized under fluorescent microscopy (Nikon ECLIPSE TE2000-U; Nikon Instruments Inc, Melville, NY). Images were captured using a Digital Eclipse DXM1200 digital camera (Nikon Instruments Inc). For the determination of intracellular $\mathrm{Pt}$ content, cells were scraped off the dish after 16 hours of treatment with various forms of carboplatin and were pelleted at $1200 \mathrm{rpm}$ for 3 minutes. The cells were washed at least twice with PBS before performing a cell count with trypan blue. Subsequently, $1 \times 10^{5}$ cells were digested in $50 \mu \mathrm{L}$ of concentrated nitric acid by heating at $85^{\circ} \mathrm{C}$ for 1 hour. Cell debris was removed by centrifugation at 13,000 rpm for 15 minutes. The resultant sample was then diluted four times with double-distilled water before the determination of Pt content by AAS.

\section{In vitro cytotoxicity assay}

Approximately 5000 cells per well were seeded in 96-well plates and allowed to attach overnight. On the following day, $200 \mu \mathrm{L}$ of fresh medium alone or containing the required concentrations of carboplatin in free or liposome forms was added to the cells. The plates were subsequently incubated at $37^{\circ} \mathrm{C}$ for 72 hours. At the end of this incubation period, $50 \mu \mathrm{L}$ of MTT in media $(1 \mathrm{mg} / \mathrm{mL})$ was added to the cells and they were incubated for a further 4 hours. Finally, the precipitated formazan was solubilized in $150 \mu \mathrm{L}$ of dimethyl sulfoxide, and absorbance was read at $570 \mathrm{~nm}$ using a plate reader (Tecan Infinite ${ }^{\circledR}$ M2000; Tecan Group Ltd, Mannedorf, Switzerland). Cancer cell viability was calculated from the absorbance values based on the following equation:

$$
\text { Viability }=\frac{\mathrm{Abs}_{\text {test }}-\mathrm{Abs}_{\text {blank }}}{\mathrm{Abs}_{\text {control }}-\mathrm{Abs}_{\text {blank }}} \times 100 \%,
$$

where $\mathrm{Abs}_{\text {test }}, \mathrm{Abs}_{\text {blank }}$, and $\mathrm{Abs}_{\text {control }}$ represent the absorbance readings from the drug-treated cells, wells without cells (media alone), and the untreated cells, respectively. The $\mathrm{IC}_{50}$ values were estimated from the MTT viability data using CalcuSyn software (v 3.0; Biosoft, Cambridge, UK).

\section{In vivo efficacy study}

Twenty-four female severe combined immunodeficiency mice were inoculated intraperitoneally with $5 \times 10^{6}$ IGROV-1 cells. ${ }^{22}$ After 14 days of tumor inoculation, the mice were randomly divided into four groups (six per group) and injected intraperitoneally with (1) saline, (2) free carboplatin, (3) carboplatin-loaded NT liposomes, or (4) carboplatin-loaded FRT liposomes. Carboplatin was administered at a dose of $15 \mathrm{mg} / \mathrm{kg}$ and following a schedule of twice-weekly administration (on Monday and Thursday) for a total of 3 weeks (ie, on days 14, 18, 21, 25, 28, and 32). Body weight of the mice was monitored at least three times a week from the date of arrival of the animals until the start of experiment, and then daily during treatment with carboplatin; this was in accordance with Institutional Animal Care and Use Committee-approved protocol. All mice were monitored daily for signs of decrease in physical activity, pain, and disease progression. Severely ill animals or animals showing weight loss or gain in excess of $20 \%$ were euthanized with an overdose of inhaled carbon dioxide. Mice surviving till the end of the experiment were termed long-term survivors and were euthanized on day 60 .

All mice undergoing efficacy study were subjected to blood collection 24 hours after doses 2, 4, and 6. Briefly, $50 \mu \mathrm{L}$ of blood was collected from each mouse in heparincontaining tubes by facial vein bleeding technique. This nonterminal procedure allows repeated sampling using alternate sides of the face (at the area of the mandible). The blood sample contains a mixture of venous and arterial blood. Plasma was obtained by centrifugation at 13,000 rpm for 15 minutes and was stored at $-80^{\circ} \mathrm{C}$ until analysis. Plasma Pt content was determined by AAS according to an established procedure. ${ }^{23}$ The plasma samples were mixed with concentrated nitric acid and heated at $85^{\circ} \mathrm{C}$ for 1 hour. Debris was pelleted by centrifugation at $13,000 \mathrm{rpm}$ for 15 minutes, and the resultant supernatant was diluted with double-distilled water for AAS analysis, as previously described.

\section{Histopathological evaluation of tissue sections}

Lungs, liver, and kidneys were excised from all groups of mice undergoing chemotherapy for histopathological evaluation of the tissue samples. The tissues were fixed in 
$10 \%$ buffered formalin solution, embedded in paraffin, and subsequently sectioned at $4 \mu \mathrm{m}$ for hematoxylin and eosin staining. Tissues from at least three mice per group were stained and analyzed by a board-certified veterinary pathologist blinded to the study.

\section{Statistics}

Unless specified otherwise, results are expressed as the mean plus or minus the standard error of the mean from three independent experiments. Statistical analyses were performed with one-way analysis of variance and the post hoc Newman-Keuls test using GraphPad Prism ${ }^{\circledR}$ software (v 2.00; GraphPad Software Inc, San Diego, CA). Survival curves were estimated using the Kaplan-Meier technique through MedCalc ${ }^{\circledR}$ statistical software (v 11.4.4.0; MedCalc Software, Mariakerke, Belgium). A log-rank test for comparisons was used when required. A $P$-value of $<0.05$ was considered to be statistically significant.

\section{Results}

\section{Carboplatin encapsulation into NT and FRT liposomes}

As the first step, encapsulation of carboplatin into liposomes was optimized based on the bulk lipid component. Two saturated lipids, DPPC and DSPC, were chosen, as the use of these lipids would yield gel-phase lipid bilayers at physiological temperatures that promote stable drug encapsulation. Carboplatin was encapsulated into the liposomes using the passive equilibration method described by Woo et al. ${ }^{20}$ The initial $\mathrm{D} / \mathrm{L}$ weight ratio used for carboplatin encapsulation was $0.25: 1$, and the liposomal lipid concentration was $80 \mathrm{mg} / \mathrm{mL}$ with a drug-loading temperature of $55^{\circ} \mathrm{C}$. As demonstrated in Figure 1A, encapsulation of carboplatin occurred within 15 minutes of incubation, and maximum loading was achieved at 60 minutes for the different formulations. The final $\mathrm{D} / \mathrm{L}$ weight ratios achieved in the DPPC-NT and the DSPC-NT liposomes were $0.062 \pm 0.004$ and $0.040 \pm 0.006$, respectively. Similar drug loading was achieved in DPPC-FRT and DSPC-FRT, with final $\mathrm{D} / \mathrm{L}$ weight ratios of $0.057 \pm 0.006$ and $0.036 \pm 0.003$, respectively. To examine how efficient the drug encapsulation procedure was, drug encapsulation efficiencies for DPPC-NT, DPPC-FRT, DSPC-NT, and DSPC-FRT liposomes were calculated and found to be $25 \%, 23 \%, 16 \%$, and $14 \%$, respectively. DPPC-based liposomes appeared to have slightly higher drug encapsulation than DSPC-based

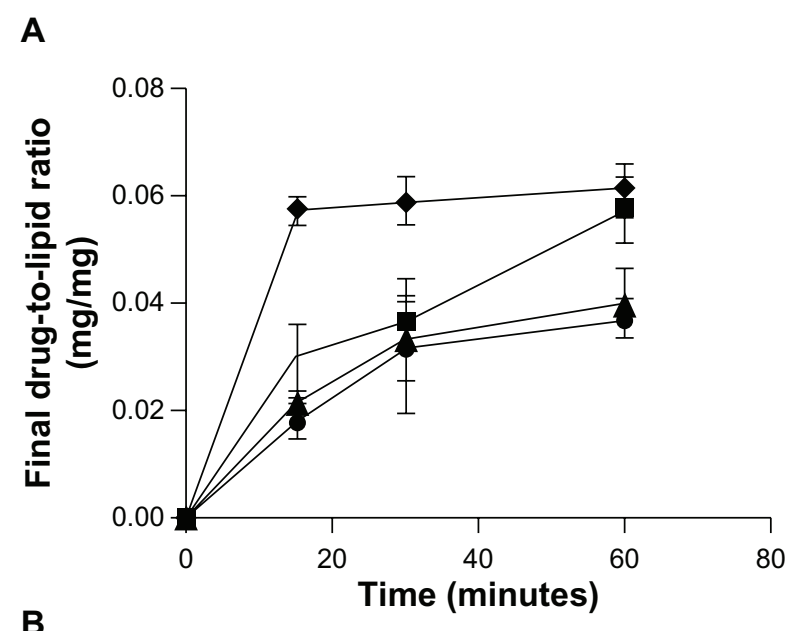

B
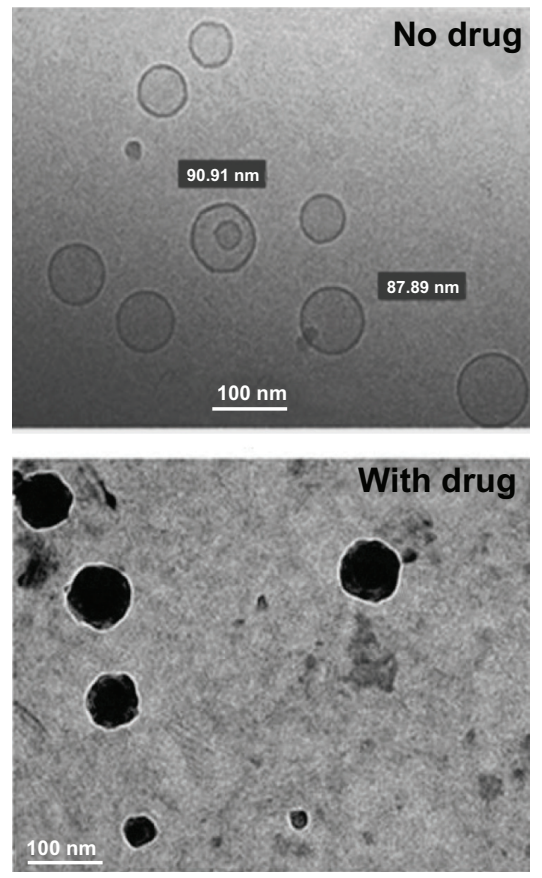

Figure I (A) Time course of carboplatin loading by passive equilibration method into different liposomes; (B) representative cryogenic transmission electron microscopy images of empty and carboplatin-loaded liposomes* from at least three liposome batches.

Notes: Results shown are the mean plus or minus the standard error of the mean obtained from at least three independent experiments; $=$ DPPC/DSPE-PEG $_{1000}[\mathrm{molar}$ ratio, 95:5], or DPPC-NT); * = DPPC/DSPE-PEG 1000 /DSPE-PEG 2000 -folate [molar ratio, 95:4.8:0.2], or DPPC-FRT); $\boldsymbol{\Delta}$ = DSPC/DSPE-PEG 1000 [molar ratio, 95:5], or DSPC-NT); $\bullet=$ DSPC/DSPE-PEG $_{1000}$ /DSPE-PEG ${ }_{2000}$-folate (molar ratio, 95:4.8:0.2; DSPC-FRT).

liposomes, although the difference was not statistically significant $(P>0.05)$.

The mean size of all liposome formulations, regardless of carboplatin encapsulation, was in the range of $100-110 \mathrm{~nm}$, with polydispersity indices of $<0.15$, as determined by the Zetasizer. Cryo-TEM was performed to further characterize the morphology of empty and carboplatin-loaded DPPC-FRT liposomes (Figure 1B). As 
carboplatin is a Pt-based drug with high electron density, ${ }^{20}$ substantial difference in the cryo-TEM images between empty and drug-loaded liposomes was anticipated. Indeed, carboplatin-loaded liposome showed a dense core, which was absent in empty liposome. Carboplatin is likely to be precipitated after loading into liposomes, with the sample cooled to room temperature. This is supported by calculating the concentration of liposome-associated carboplatin, which was found to be $45 \mathrm{mg} / \mathrm{mL}$ based on the final $\mathrm{D} / \mathrm{L}$ weight ratio of $0.057: 1$, a trap volume of $1 \mu \mathrm{L} / \mu \mathrm{mol}$ lipid for the $100 \mathrm{~nm}$ DPPC-FRT liposome, and the water solubility of carboplatin of $14 \mathrm{mg} / \mathrm{mL} .{ }^{20}$

\section{In vitro drug release from NT and FRT liposomes}

The in vitro drug release profiles were obtained for DPPC-NT, DPPC-FRT, DSPC-NT, and DSPC-FRT liposomes, based on the dialysis method against a sink of $1: 1000 \mathrm{v} / \mathrm{v}$ PBS at $37^{\circ} \mathrm{C}$ (Figure 2A). All drug release profiles were similar, and they did not indicate any significant difference between the NT and FRT formulations at different time points $(P>0.05)$. A similar study was performed in the presence of $50 \%$ FBS at $37^{\circ} \mathrm{C}$ (Figure 2B), and the results showed a similar trend - no statistical significant difference was seen when comparing NT and FRT liposomes. However, carboplatin was released faster from the liposomes in serum-containing medium than PBS alone.

Since DPPC-based liposomes (DPPC-FRT and DPPCNT) showed higher drug encapsulation than DSPC-based liposomes, and since they did not show any significant difference in drug release pattern, all further studies were performed with DPPC-FRT and DPPC-NT liposomes. Henceforth, DPPC-NT and DPPC-FRT liposomes will be termed NT and FRT liposomes, respectively, unless otherwise specified.

\section{Intracellular accumulation of carboplatin in free and liposomal forms}

For an anticancer drug such as carboplatin to exert its biological activity, it is crucial for the drug to be delivered intracellularly. As such, the next set of experiments in the current study aimed to probe the intracellular accumulation of carboplatin in free and liposomal forms. The accumulation of the NT and FRT liposomes was first probed in the FRoverexpressing IGROV-1 ovarian cancer cell line, whereby the liposomes were tagged with the fluorescent lipid DiI. FRT liposomes (Figure 3A, panel i) showed much higher cellular accumulation than NT liposomes (Figure 3A, panel ii), and
A
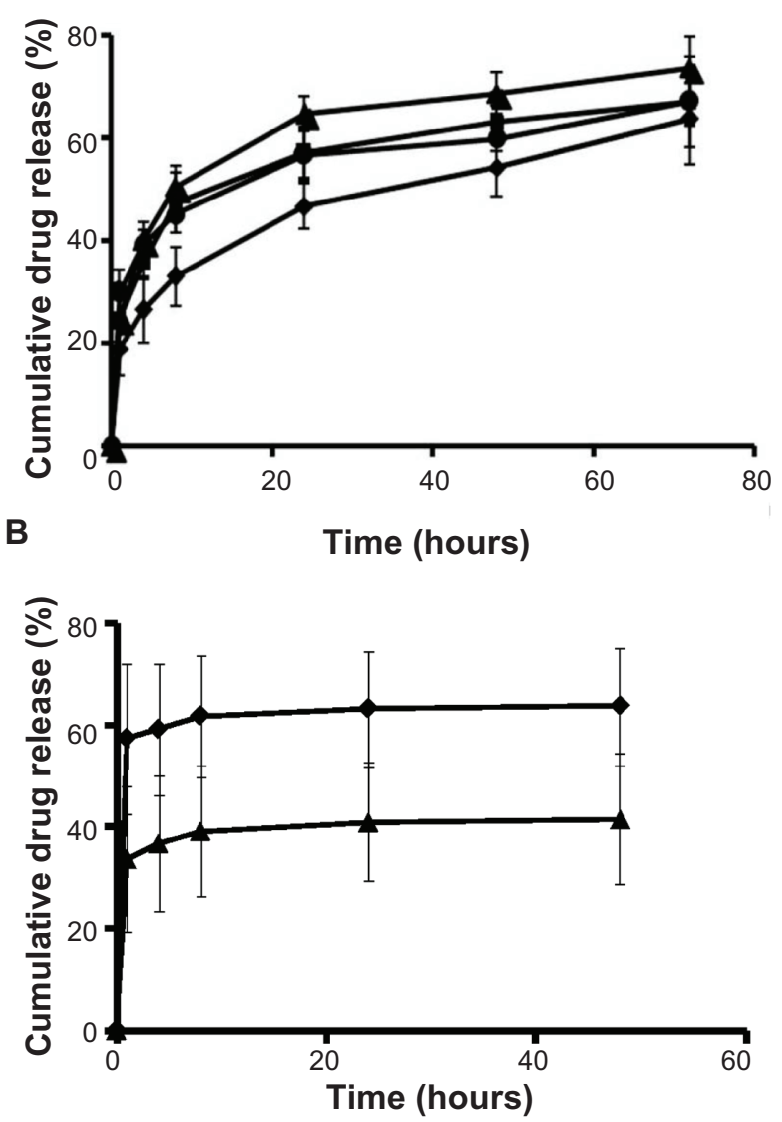

Figure 2 Cumulative release of carboplatin from various liposomes at $37^{\circ} \mathrm{C}$ under sink conditions (I:I000 v/v) in (A) phosphate-buffered saline and (B) $50 \%(\mathrm{v} / \mathrm{v})$ fetal bovine serum.

Notes: Results shown are the mean plus or minus the standard error of the mean obtained from at least three independent experiments; $\$$ DPPC/DSPE-PEG 1000 [molar ratio, 95:5], or DPPC-NT); = DPPC/DSPE-PEG ${ }_{1000} / \mathrm{DSPE}^{-P E \mathrm{DE}_{200}}$-folate [molar ratio, 95:4.8:0.2], or DPPC-FRT); $\boldsymbol{\Delta}$ = DSPC/DSPE-PEG ${ }_{1000}$ [molar ratio, 95:5], or DSPC-NT); $\bullet=\mathrm{DSPC} \mathrm{DSPE}_{-\mathrm{PEG}}{ }_{1000} /$ DSPE-PEG $_{2000}$-folate (molar ratio, 95:4.8:0.2; DSPC-FRT).

in the presence of $1 \mathrm{mM}$ of free folic acid, the accumulation of the FRT liposomes was completely blocked (Figure 3A, panel iii). Furthermore, Pt concentration in IGROV-1 cancer cells was determined and compared among the three formulations of carboplatin. As shown in Figure 3B, the intracellular Pt level in IGROV-1 cells treated with FRT liposomes was approximately twofold higher than those treated with free carboplatin or NT carboplatin liposomes, which was statistically significant $(P<0.05)$.

\section{Viability of ovarian cancer cells upon exposure to carboplatin in free or liposomal forms}

Figure 4 illustrates the concentration-dependent reduction in IGROV-1 cell viability upon exposure to carboplatin as free drug or as delivered via NT and FRT liposomes. 
A
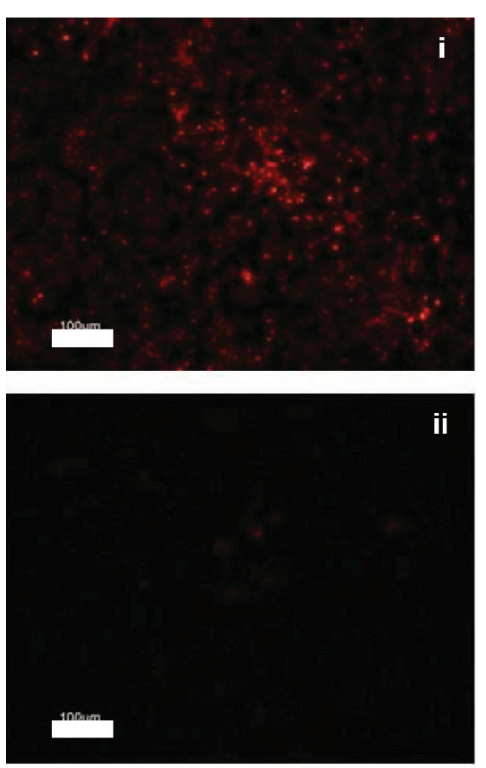

iii

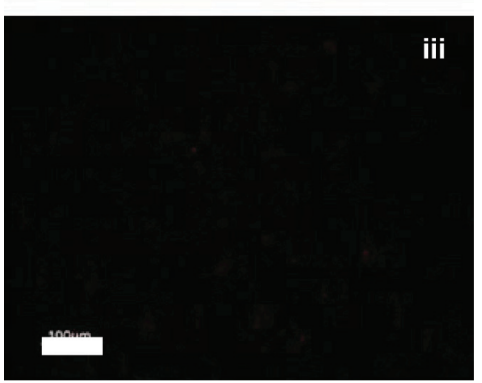

B

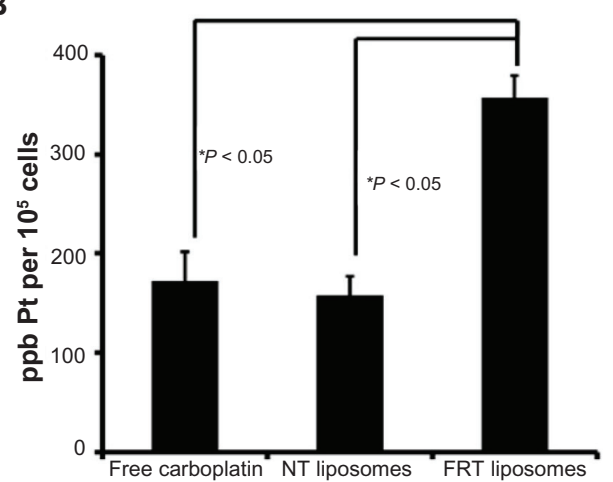

Figure 3 (A) Cellular accumulation of Dil-labeled folate receptor-targeted (FRT) liposomes (i), nontargeted (NT) liposomes (ii), and FRT liposomes in the presence of I mM of free folic acid (iii) in IGROV-I cells after 24 hours. (B) Cellular platinum (Pt) content in IGROV-I cells after treatment with free carboplatin, carboplatinloaded NT liposomes, or carboplatin-loaded FRT liposomes.

Notes: Images are representative of three independent experiments; data represent the mean plus or minus the standard error of the mean obtained from three independent experiments.

Abbreviation: ppb, parts per billion.

Cell viability was also examined in cells treated with FRT carboplatin liposomes in the presence of $1 \mathrm{mM}$ of free folic acid. Based on the cell viability data, the $\mathrm{IC}_{50}$ values for free carboplatin, NT carboplatin liposomes, and FRT carboplatin liposomes were estimated and found to be $24.0 \pm 1.6 \mu \mathrm{M}$, $40.1 \pm 5.0 \mu \mathrm{M}$, and $13.1 \pm 0.6 \mu \mathrm{M}$, respectively, with FRT

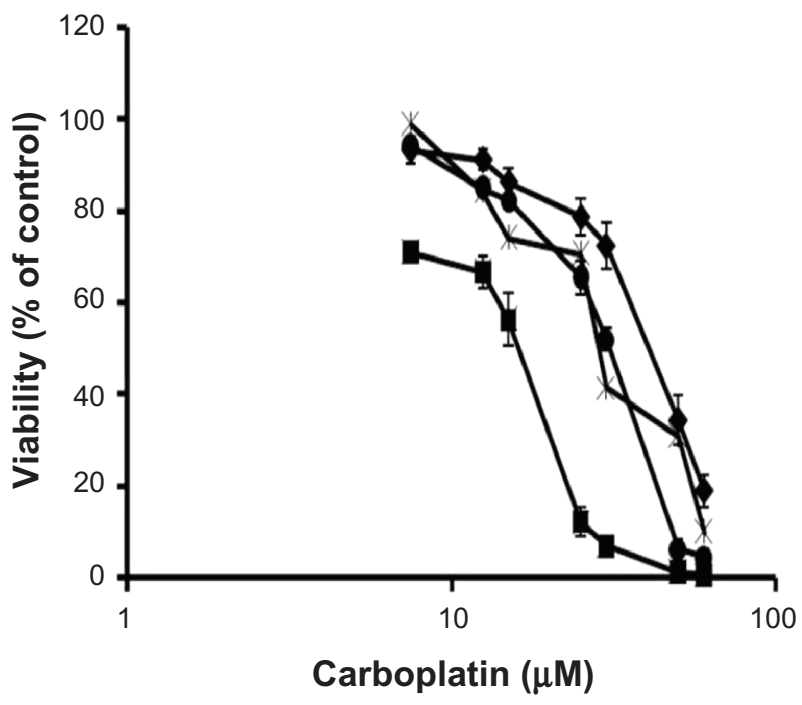

Figure 4 Viability of IGROV-I cells upon 72-hour exposure to free carboplatin (•), carboplatin-loaded nontargeted liposome $(\downarrow)$, carboplatin-loaded folate receptortargeted liposome ( $\boldsymbol{\nabla})$, and carboplatin-loaded folate receptor-targeted liposome with I mM folic acid $(\times)$.

Note: Data represent the mean plus or minus the standard error of the mean obtained from five independent experiments.

liposomes significantly more effective in reducing cell viability (Table 1). When $1 \mathrm{mM}$ of free folic acid was added to the FRT carboplatin treatment, the $\mathrm{IC}_{50}$ value was significantly increased - from $13.1 \pm 0.6 \mu \mathrm{M}$ to $29.0 \pm 5.3 \mu \mathrm{M}$. Of note, drug-free NT liposomes and FRT liposomes did not show any cytotoxicity towards IGROV-1 cells, with viability of $98.9 \% \pm 3.4 \%$ and $99.1 \% \pm 0.3 \%$, respectively, at the highest liposomal lipid concentration of $500 \mu \mathrm{M}$. This finding is in agreement with a previous report that empty FRT liposomes were nontoxic to the animals and did not contribute to the anticancer activity. ${ }^{24}$ Collectively, the current study's in vitro results are in agreement with the notion that FR-mediated endocytosis of FRT liposome-encapsulated drug could enhance the intracellular accumulation as well as the potency of the encapsulated drug.

Table I IC IC $_{50}$ values of free carboplatin, carboplatin-loaded nontargeted (NT) liposomes, and carboplatin-loaded folate receptor-targeted (FRT) carboplatin liposomes in IGROV-I cells

\begin{tabular}{ll}
\hline Group & IC $_{50}\left(\mu \mathbf{M}^{\mathrm{a}}\right.$ \\
\hline Free drug & $24.0 \pm 1.6^{\mathrm{b}, \mathrm{c}}$ \\
Carboplatin-loaded NT liposomes & $40.1 \pm 5.0^{\mathrm{b}, \mathrm{c}}$ \\
Carboplatin-loaded FRT liposomes & $13.1 \pm 0.6^{\mathrm{c}, \mathrm{d}}$ \\
Carboplatin-loaded FRT liposomes + I mM free folic acid & $29.0 \pm 5.3^{\mathrm{d}}$ \\
\hline
\end{tabular}

Notes: ${ }^{a}$ Results shown are the mean plus or minus the standard error of the mean obtained from at least three independent experiments; ${ }^{\mathrm{b}} \mathrm{P}<0.05$, free drug versus NT liposomes; ${ }^{c} p<0.05$, FRT liposomes versus free drug and NT liposomes; ${ }^{d} P<0.05$, FRT liposomes with versus without I mM free folic acid. 


\section{In vivo therapeutic efficacy of carboplatin in free and liposomal forms}

The therapeutic efficacy of carboplatin delivered in free or liposomal forms was investigated in the intraperitoneally grown human IGROV-1 ovarian tumor xenograft model, which was selected to mimic the clinical nature of tumor burden in women with advanced-stage ovarian cancer and FR overexpression..$^{22}$ Carboplatin was given at $15 \mathrm{mg} / \mathrm{kg}$ and following a schedule of two doses per week (on Monday and Thursday) for a total of 3 weeks. Kaplan-Meier survival curves are presented in Figure 5A, and the survival data are summarized in Table 2. Mice in the saline control, free

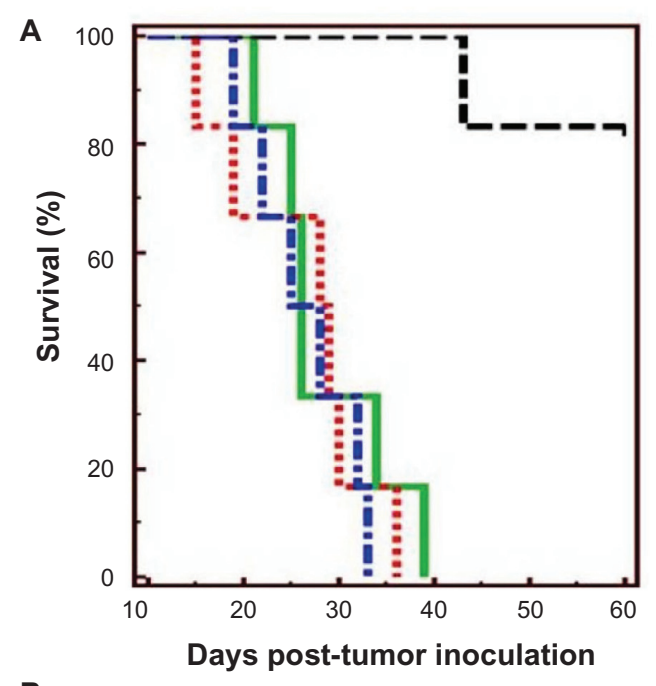

B

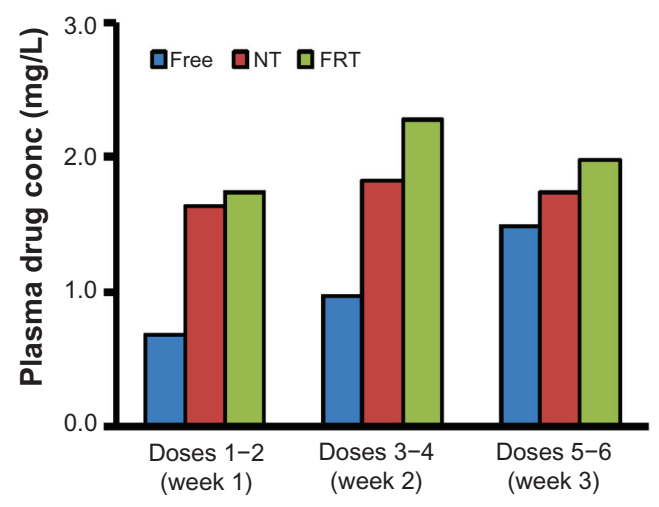

Figure 5 (A) Kaplan-Meier survival curves of mice bearing intraperitoneal IGROV-I ovarian tumor xenograft treated with saline (blue), free carboplatin (green), carboplatin-loaded nontargeted (NT) liposomes (red) and carboplatin-loaded folate receptor-targeted (FRT) liposomes (black). Each study group comprised six animals. (B) Plasma drug concentrations (conc) in animals treated with various intraperitoneal carboplatin formulations at a dose of $15 \mathrm{mg} / \mathrm{kg}$. Carboplatin was administered for a total of six doses, and plasma samples were collected 24 hours after doses 2, 4, and 6 .

Notes: Results shown in panel (B) were obtained from at least three mice. The exception to this was dose 6 , with $n=2$ and $n=1$ for free drug and carboplatinloaded NT liposome groups, respectively. carboplatin, and NT carboplatin liposome groups did not have any survivors at the end of the study period (60 days post-tumor inoculation), and all died within 19-39 days posttumor inoculation. All dead mice were autopsied to confirm that they had died from tumor burden. The median survival time for the saline control, free drug, and NT carboplatin liposome groups was $26.5,26$, and 28.5 days, respectively. In contrast, five out of six mice treated with FRT carboplatin liposomes survived until the end of the study, with a survival rate of $83 \%$. A log-rank test indicated a statistically significant difference between the FRT carboplatin liposome group and the saline control, free drug, and NT carboplatin liposome groups $(P<0.01)$.

Previous studies have shown that IP administration of doxorubicin encapsulated in FRT and NT liposomes led to a longer circulation lifetime than free doxorubicin. ${ }^{24}$ In light of this finding, plasma Pt concentration in the current study was determined each week during the 3-week treatment period to estimate the level of carboplatin in the systemic circulation. Plasma samples were collected 24 hours after doses 2, 4, and 6. As shown in Figure 5B, the plasma Pt concentration of the free drug group was approximately twofold lower than the concentrations of the NT and FRT carboplatin liposome groups during the first and second weeks of treatment, although the difference was not statistically significant $(P>0.05)$.

Changes in body weight of the animals were monitored over the study period of 60 days as a gross indicator to the well-being of the animals under the experimental conditions (Supplementary figure 1). Acute weight loss could not be observed in any of the treated groups during the course of study. However, the percent weight change at nadir for the NT carboplatin liposome group was found to be $\sim 20 \%$, although the difference between this and the other groups was not statistically significant (Table 2). The saline control group demonstrated an increase in body weight, which could have been due to the growing tumor and the accumulation of ascitic fluid. Of note, the FRT carboplatin liposome group did not show any significant change in body weight over the 60-day study period $(P>0.05)$, which is a similar result to that observed in the normal, healthy mice (without tumor or drug treatment). Future animal toxicological studies are necessary to fully elucidate the toxicity profile of the FRT carboplatin liposome formulation, involving the evaluation of blood chemistry (such as alanine aminotransferase, aspartate aminotransferase, alkaline phosphatase, bilirubin, creatinine, urea, and major electrolytes) and histological evaluation of vital organs (liver, gallbladder, spleen, lung, kidney, heart, 
Table 2 In vivo efficacy of intraperitoneal administration of carboplatin in various forms in intraperitoneal IGROV-I ovarian tumor xenograft

\begin{tabular}{llllc}
\hline Study group & $\begin{array}{l}\text { Median survival } \\
\text { time (days) }\end{array}$ & $\begin{array}{l}\text { Survival time } \\
\text { (range in days) }\end{array}$ & $\begin{array}{l}\text { Long-term } \\
\text { survivor }\end{array}$ & $\begin{array}{l}\text { Weight change } \\
\text { at nadir (\%) }\end{array}$ \\
\hline Saline control & 26.5 & $19-33$ & $0 / 6$ & $+4.1 \pm 1.6$ \\
Free drug & 26 & $21-39$ & $0 / 6$ & $-9.3 \pm 7.6$ \\
NT liposomes & 28.5 & $15-36$ & $0 / 6$ & $-20.5 \pm 10.4$ \\
FRT liposomes & NA & 43 to $>60$ & $5 / 6$ & $-8.0 \pm 1.4$ \\
\hline
\end{tabular}

Notes: a $P<0.0$ I by log-rank test; blong-term survivor is defined as an animal surviving until the end of the experiment (duration 60 days post-tumor inoculation); ${ }^{c}$ weight change at nadir $(\%)=$ (weight at nadir - weight before treatment $) /$ weight before treatment $\times 100 \%$.

Abbreviations: FRT, folate receptor-targeted; NA, not applicable; NT, nontargeted.

intestine, lymph nodes, marrow, bladder, and brain) under acute and chronic settings.

\section{Histopathological evaluation on tissues from treated animals}

In addition to monitoring the survival of the animals, histopathological evaluation of tissues collected from the control animals and from those treated with various forms of carboplatin was performed to probe the presence of tumor cells, so as to reflect disease progression and therapeutic efficacy. It has been established that ovarian cancer cells can invade major organs such as the lungs and liver through the lymphatic system. ${ }^{1}$ This phenomenon could be seen in the IGROV-1 IP tumor xenograft model, whereby tumor cells were present in the lungs and livers of saline-treated tumor-bearing animals (Figure 6, A panels), with the mediastinal lymph node filled with tumor cells (Supplementary figure 2).

As shown in Figure 6, substantial metastasis of the tumor cells could be observed in the lungs obtained from the free carboplatin and NT carboplatin liposome groups. Tumor cells were found within the capillaries of the lungs as well as the lung lobes. In contrast, metastasis could not be observed in the long-term survivors treated with FRT carboplatin liposomes. A similar trend was observed in the liver sections from the treated animals, whereby clusters of tumor cells were present on the outer surface of the liver capsule in the saline control, free carboplatin, and NT carboplatin liposome groups but not in the FRT carboplatin liposome group. These results suggest that the FRT carboplatin liposome formulation was effective in treating advanced metastatic ovarian cancer.

\section{Discussion}

Recent clinical trials have shed light on the usefulness of IP administration of Pt-based compounds to extend the overall survival of ovarian cancer patients. ${ }^{7,825}$ As mentioned, IP Pt-based therapy provides high locoregional concentrations of the drug and longer exposure in the peritoneal cavity as compared with the intravenous route. ${ }^{5}$ Yet, the high local drug concentration would potentially give rise to higher risk of toxicities. ${ }^{5}$ In the current study, the authors have further improved the approach of the IP carboplatin regimen by coupling this route of drug administration with a FRT liposomal system as the enabling technology for achieving intracellular delivery of carboplatin into the ovarian cancer cells, increasing the therapeutic efficacy. The in vitro and in vivo data demonstrated significant improvement in the therapeutic efficacy of carboplatin through the use of FRT liposomes in a metastatic FR-positive ovarian tumor xenograft model. The biological performance of the FRT carboplatin liposomes will be discussed in light of the formulation attributes.

With farletuzumab, the anti-FR monoclonal antibody, emerging as a promising targeted therapy for ovarian cancer, ${ }^{18,19}$ the development of therapies targeting the human FR would continue to attract much attention. Over the years, various research groups have developed FRT liposomes for different conventional chemotherapeutics, with the aim of increasing the therapeutic efficacy of the encapsulated drug. ${ }^{26,27}$ Nevertheless, limited studies are available for the evaluation of FRT liposome-based anticancer drug therapies in FR-overexpressing ovarian cancer cells/models. In view of the therapeutic promise of IP Pt-based drug administration and the emerging importance of FRT therapy in the treatment of ovarian cancer, the authors' approach of IP administration of FRT carboplatin liposome formulation is the first to demonstrate the feasibility of such a therapeutic strategy that is closely in line with the anatomical disease site and clinically relevant.

Various factors could contribute to the superior therapeutic efficacy of FRT carboplatin liposomes, and the in vitro and in vivo data argue in favor of increased intracellular delivery of carboplatin through a FRT liposomal system. As evidenced by Figures 3 and 4, FRT liposomes could significantly increase the accumulation of carboplatin and thus its potency 


\section{Lung tissue}

A

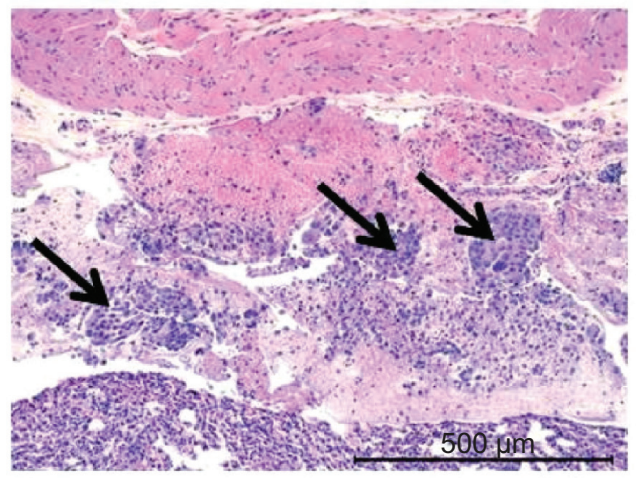

C

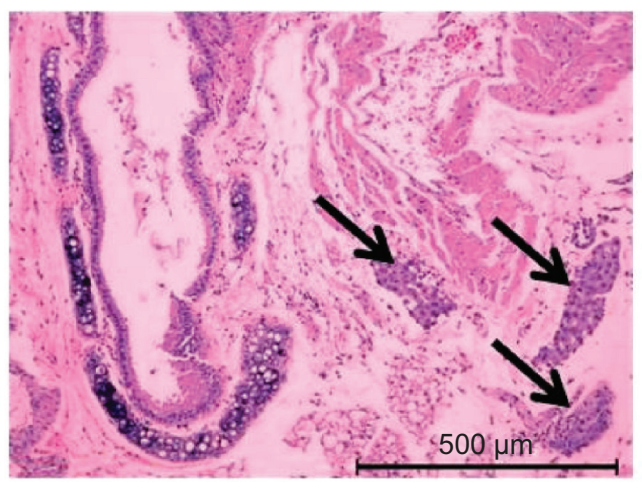

B

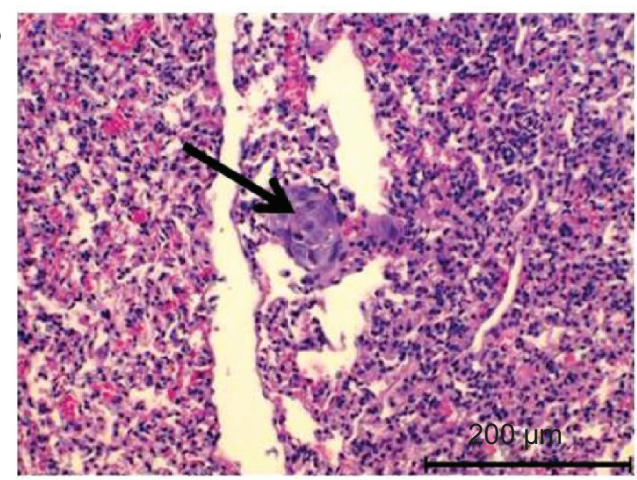

D

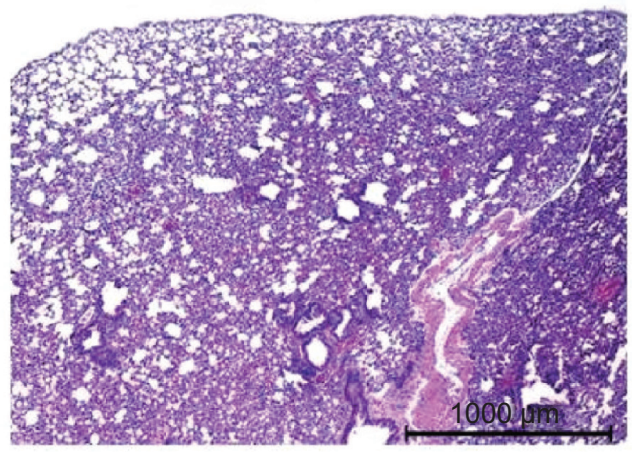

\section{Liver tissue}

A

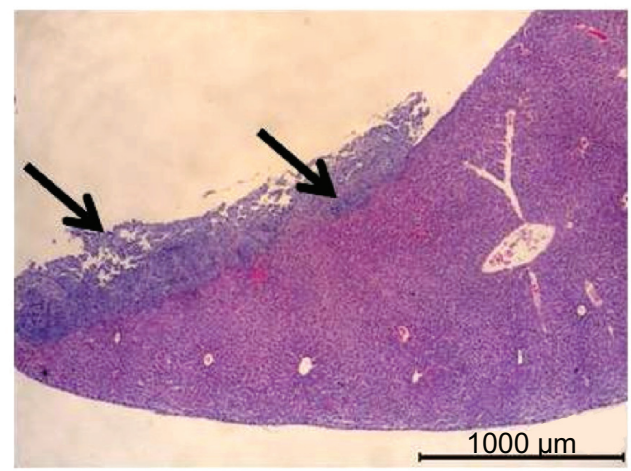

C

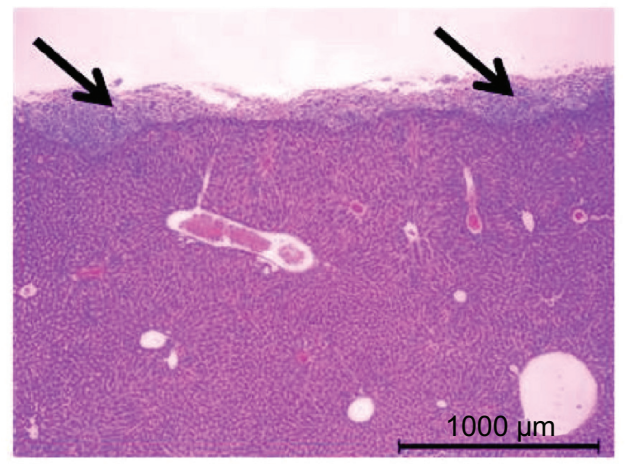

B

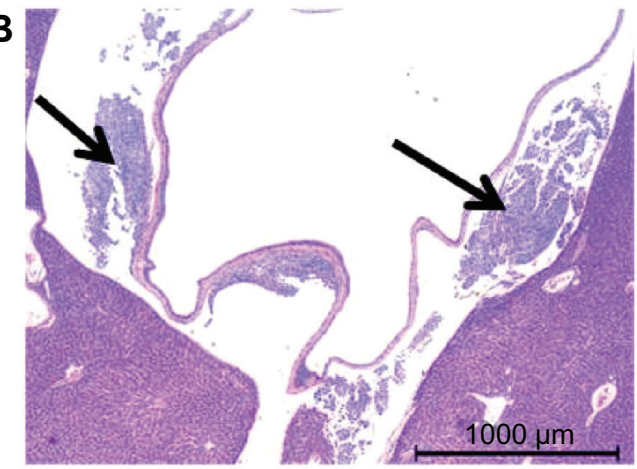

D

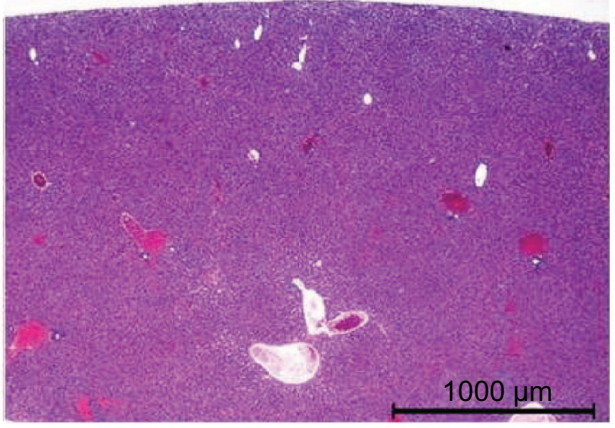

Figure 6 Histopathological evaluation of excised lung tissue (top) and liver tissue (bottom) indicating the presence of cancer cells in mice treated with (A) saline, (B) free carboplatin, and $(\mathbf{C})$ carboplatin-loaded nontargeted liposome. Mice treated with carboplatin-loaded folate receptor-targeted liposome (D) that survived the study period did not show any presence of cancer cells.

Notes: Representative images are shown from at least three animals, with arrows indicating the presence of cancer cells. 
in the IGROV-1 ovarian cancer cells. An earlier study that demonstrated enhanced accumulation of fluorescent FRT liposomes (non-drug loaded), as compared with NT liposomes, in IGROV-1 cells and tumor-associated macrophages from mice bearing IP IGROV-1 tumor xenograft provides further evidence to support the efficacy findings of the current study. ${ }^{22}$ Of importance, it is unlikely that the difference in the in vivo therapeutic outcome between the NT and FRT liposome formulations could be attributed to the drug release properties of these two formulations, as both NT and FRT liposomes have similar drug release profiles (Figure 2).

The IP IGROV-1 ovarian tumor xenograft model was chosen to mimic the metastatic stage of ovarian cancer, and IP therapy will allow systemic drug exposure in the extraperitoneal tumor sites after drug absorption. Comparing the plasma carboplatin concentrations of the three forms of carboplatin during the course of the efficacy study, systemic carboplatin exposure was not significantly different among the three formulations, albeit NT and FRT liposomes yielded an approximately twofold higher Pt concentration in plasma (Figure 5B). Thus, increased systemic exposure to carboplatin via FRT liposome delivery is not likely to be a contributing factor to the increased therapeutic activity of this formulation. Suboptimal cellular accumulation of carboplatin with NT liposomes and free carboplatin is likely to have resulted in poor suppression of primary tumor growth and metastasis.

The FRT carboplatin liposomes were administered at a cumulative drug dose of $90 \mathrm{mg} / \mathrm{kg}$, which was twofold lower than the cumulative maximum tolerated dose of carboplatin in mice (reported to be $180 \mathrm{mg} / \mathrm{kg}$ for the IP route). ${ }^{28}$ Other studies have used lower IP doses of carboplatin in the free or liposomal form, at a single dose of $75-85 \mathrm{mg} / \mathrm{kg},{ }^{29,30}$ which could still give rise to significantly high toxicity in the case of NT liposomal carboplatin..$^{29}$ However, the FRT carboplatin liposomes have yielded significant improvement in therapeutic activity compared with free drug and NT liposomes.

\section{Conclusion}

The results of this study demonstrate the potential of FRT carboplatin liposomes to be administered to patients at a lower dose while maintaining therapeutic efficacy with reduced risk of regimen-related toxicities. Future studies involving optimizing the dose and frequency of the FRT carboplatin liposomes, together with full animal toxicological evaluation, could facilitate the identification of a regimen that yields maximal therapeutic efficacy without drug-related toxicities.

\section{Acknowledgments}

This project is supported by a grant from the National Medical Research Council of Singapore (grant No NMRC/1109/2007). The authors thank Professor Larry H Matherly (Wayne State University School of Medicine, Detroit, MI) for providing the IGROV-1 cell line. The authors also thank the histopathology unit of the Biopolis Shared Facilities of the Agency for Science, Technology and Research, Singapore, for performing the hematoxylin and eosin staining.

\section{Disclosure}

The authors report no conflicts of interest in this work.

\section{References}

1. Aletti GD, Podratz KC, Cliby WA, Gostout BS. Stage IV ovarian cancer: disease site-specific rationale for postoperative treatment. Gynecol Oncol. 2009;112(1):22-27.

2. Barnes MN, Grizzle WE, Grubbs CJ, Partridge EE. Paradigms for primary prevention of ovarian carcinoma. CA Cancer J Clin. 2002; 52(4):216-225.

3. Au JL, Jang SH, Wientjes MG. Clinical aspects of drug delivery to tumors. J Control Release. 2002;78(1-3):81-95.

4. Lu Z, Wang J, Wientjes MG, Au JL. Intraperitoneal therapy for peritoneal cancer. Future Oncol. 2010;6(10):1625-1641.

5. Echarri Gonzalez MJ, Green R, Muggia FM. Intraperitoneal drug delivery for ovarian cancer: why, how, who, what, and when? Oncology (Williston Park). 2011;25(2):156-165, 170.

6. Armstrong DK, Bundy B, Wenzel L, et al. Intraperitoneal cisplatin and paclitaxel in ovarian cancer. $N$ Engl J Med. 2006;354(1):34-43.

7. Alberts DS, Liu PY, Hannigan EV, et al. Intraperitoneal cisplatin plus intravenous cyclophosphamide versus intravenous cisplatin plus intravenous cyclophosphamide for stage III ovarian cancer. $N$ Engl J Med. 1996;335(26):1950-1955.

8. Markman M, Bundy BN, Alberts DS, et al. Phase III trial of standard-dose intravenous cisplatin plus paclitaxel versus moderately high-dose carboplatin followed by intravenous paclitaxel and intraperitoneal cisplatin in small-volume stage III ovarian carcinoma: an intergroup study of the Gynecologic Oncology Group, Southwestern Oncology Group, and Eastern Cooperative Oncology Group. J Clin Oncol. 2001;19(4):1001-1007.

9. Wenzel LB, Huang HQ, Armstrong DK, Walker JL, Cella D; Gynecologic Oncology Group. Health-related quality of life during and after intraperitoneal versus intravenous chemotherapy for optimally debulked ovarian cancer: a Gynecologic Oncology Group study. J Clin Oncol. 2007;25(4):437-443.

10. Elferink F, van der Vijgh WJ, Klein I, ten Bokkel Huinink WW, Dubbelman R, McVie JG. Pharmacokinetics of carboplatin after intraperitoneal administration. Cancer Chemother Pharmacol. 1988;21(1): $57-60$.

11. Zimm S, Cleary SM, Lucas WE, et al. Phase I/pharmacokinetic study of intraperitoneal cisplatin and etoposide. Cancer Res. 1987;47(6): 1712-1716.

12. Miyagi Y, Fujiwara K, Kigawa J, et al. Intraperitoneal carboplatin infusion may be a pharmacologically more reasonable route than intravenous administration as a systemic chemotherapy: a comparative pharmacokinetic analysis of platinum using a new mathematical model after intraperitoneal vs intravenous infusion of carboplatin; a Sankai Gynecology Study Group (SGSG) study. Gynecol Oncol. 2005;99(3):591-596.

13. Fujiwara K, Markman M, Morgan M, Coleman RL. Intraperitoneal carboplatin-based chemotherapy for epithelial ovarian cancer. Gynecol Oncol. 2005;97(1):10-15. 
14. Fujiwara K, Sakuragi N, Suzuki S, et al. First-line intraperitoneal carboplatin-based chemotherapy for 165 patients with epithelial ovarian carcinoma: results of long-term follow-up. Gynecol Oncol. 2003;90(3):637-643.

15. Fujiwara K, Aotani E, Hamano T, et al. A randomized phase II/III trial of 3 weekly intraperitoneal versus intravenous carboplatin in combination with intravenous weekly dose-dense paclitaxel for newly diagnosed ovarian, fallopian tube and primary peritoneal cancer. Jpn J Clin Oncol. 2011;41(2):278-282.

16. Fujiwara K, Nagao S, Kigawa J, et al. Phase II study of intraperitoneal carboplatin with intravenous paclitaxel in patients with suboptimal residual epithelial ovarian or primary peritoneal cancer: a Sankai Gynecology Cancer Study Group study. Int J Gynecol Cancer. 2009;19(5):834-837.

17. Kelemen LE. The role of folate receptor alpha in cancer development, progression and treatment: cause, consequence or innocent bystander? Int J Cancer. 2006;119(2):243-250.

18. Spannuth WA, Sood AK, Coleman RL. Farletuzumab in epithelial ovarian carcinoma. Expert Opin Biol Ther. 2010;10(3):431-437.

19. Konner JA, Bell-McGuinn KM, Sabbatini P, et al. Farletuzumab, a humanized monoclonal antibody against folate receptor alpha, in epithelial ovarian cancer: a phase I study. Clin Cancer Res. 2010;16(21):5288-5295.

20. Woo J, Chiu GN, Karlsson G, et al. Use of a passive equilibration methodology to encapsulate cisplatin into preformed thermosensitive liposomes. Int J Pharm. 2008;349(1-2):38-46.

21. Fiske $\mathrm{CH}$, Subbarow Y. The colorimetric determination of phosphorus. J Biol Chem. 1925;66(2):375-400.

22. Turk MJ, Waters DJ, Low PS. Folate-conjugated liposomes preferentially target macrophages associated with ovarian carcinoma. Cancer Lett. 2004;213(2):165-172.
23. Bandak S, Goren D, HorowitzA, Tzemach D, Gabizon A. Pharmacological studies of cisplatin encapsulated in long-circulating liposomes in mouse tumor models. Anticancer Drugs. 1999;10(10):911-920.

24. Pan XQ, Wang H, Lee RJ. Antitumor activity of folate receptor-targeted liposomal doxorubicin in a KB oral carcinoma murine xenograft model. Pharm Res. 2003;20(3):417-422.

25. Gadducci A, Carnino F, Chiara S, et al. Intraperitoneal versus intravenous cisplatin in combination with intravenous cyclophosphamide and epidoxorubicin in optimally cytoreduced advanced epithelial ovarian cancer: a randomized trial of the Gruppo Oncologico Nord-Ovest. Gynecol Oncol. 2000;76(2):157-162.

26. Low PS, Antony AC. Folate receptor-targeted drugs for cancer and inflammatory diseases. Adv Drug Deliv Rev. 2004;56(8):1055-1058.

27. Gabizon A, Shmeeda H, Horowitz AT, Zalipsky S. Tumor cell targeting of liposome-entrapped drugs with phospholipid-anchored folic acidPEG conjugates. Adv Drug Deliv Rev. 2004;56(8):1177-1192.

28. Pratesi G, Tortoreto M, Corti C, Giardini R, Zunino F. Successful local regional therapy with topotecan of intraperitoneally growing human ovarian carcinoma xenografts. Br J Cancer. 1995;71(3):525-528.

29. Fichtner I, Reszka R, Goan SR, Naundorf H. Carboplatin-liposomes (CPL) in immunodeficient mice: improved antitumor activity for breast carcinomas and stimulation of hematopoiesis. Med Oncol. 1994;11(3-4):111-119.

30. Jandial DD, Messer K, Farshchi-Heydari S, Pu M, Howell SB. Tumor platinum concentration following intraperitoneal administration of cisplatin versus carboplatin in an ovarian cancer model. Gynecol Oncol. 2009;115(3):362-366. 


\section{Supplementary figures}

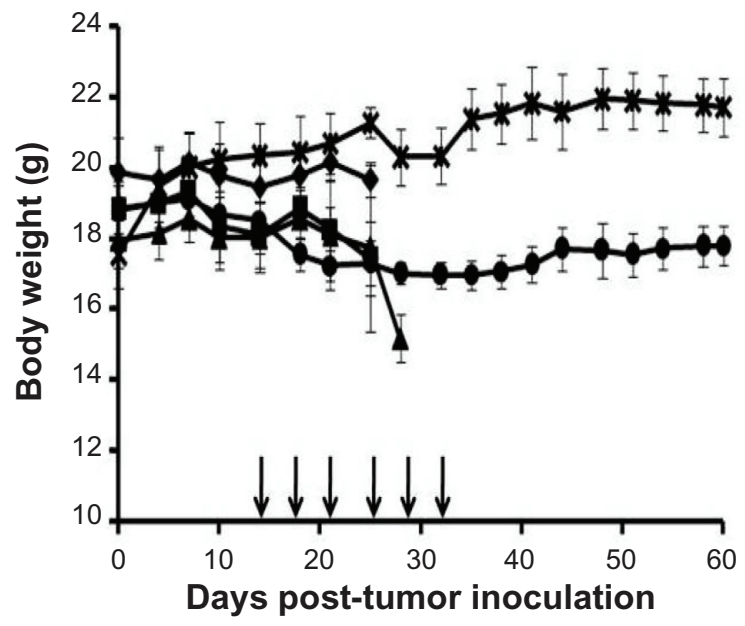

Figure SI Changes in body weight in various study groups, including saline $(\downarrow)$, free carboplatin ( $\mathbf{\square})$, carboplatin-loaded nontargeted liposome $(\mathbf{\Delta})$, carboplatin-loaded folate receptor-targeted liposme $(\bullet)$, and healthy control mice $(\times)$.

Notes: Body weights were measured at least three times a week, with the arrows indicating the intraperitoneal administration of treatment. Results shown are the mean plus or minus the standard error of the mean obtained from at least three mice.

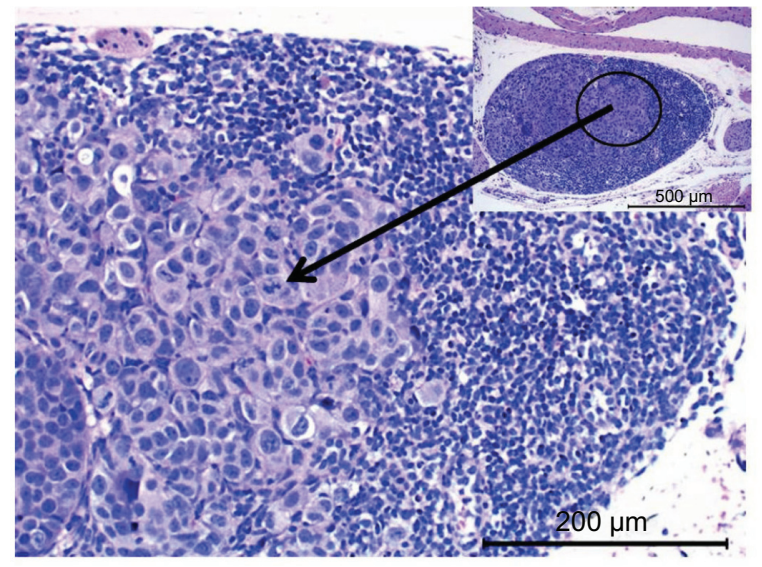

Figure S2 Histopathological evaluation indicating the presence of cancer cells in mediastinal lymph node of saline control.

Notes: Arrow indicates the presence of cancer cells. Representative image of lymph node metastasis is presented.
International Journal of Nanomedicine

\section{Publish your work in this journal}

The International Journal of Nanomedicine is an international, peerreviewed journal focusing on the application of nanotechnology in diagnostics, therapeutics, and drug delivery systems throughou the biomedical field. This journal is indexed on PubMed Central, MedLine, CAS, SciSearch ${ }^{\circledR}$, Current Contents ${ }^{\circledR} /$ Clinical Medicine,

\section{Dovepress}

Journal Citation Reports/Science Edition, EMBase, Scopus and the Elsevier Bibliographic databases. The manuscript management system is completely online and includes a very quick and fair peer-review system, which is all easy to use. Visit http://www.dovepress.com/ testimonials.php to read real quotes from published authors. 